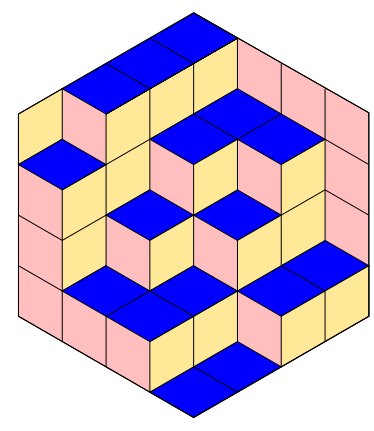

Patrick Wegener \& Sophiane Yahiatene

A note on non-reduced reflection factorizations of Coxeter elements

Volume 3, issue 2 (2020), p. 465-469.

<http://alco.centre-mersenne.org/item/ALCO_2020__3_2_465_0>

(C) The journal and the authors, 2020.

Some rights reserved.

(c) BY This article is licensed under the

Creative Commons ATtRibution 4.0 InTERnATIONAL LiCEnSE.

http://creativecommons.org/licenses/by/4.0/

Access to articles published by the journal Algebraic Combinatorics on the website http://alco.centre-mersenne.org/ implies agreement with the Terms of Use (http://alco.centre-mersenne.org/legal/).

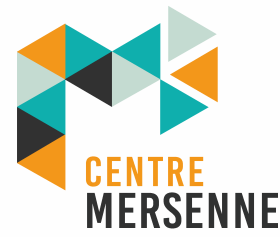

Algebraic Combinatorics is member of the Centre Mersenne for Open Scientific Publishing www.centre-mersenne.org 


\title{
A note on non-reduced reflection factorizations of Coxeter elements
}

\author{
Patrick Wegener \& Sophiane Yahiatene
}

\begin{abstract}
We extend a result of Lewis and Reiner from finite Coxeter groups to Coxeter groups of finite rank by showing that two reflection factorizations of a Coxeter element lie in the same Hurwitz orbit if and only if they share the same multiset of conjugacy classes.
\end{abstract}

\section{INTRODUCTION}

Given a Coxeter system $(W, S)$ with set of reflections $T$, the braid group (e.g. see [1] for a definition) acts on reflection factorizations of a given element $w \in W$, that is it acts on tuples $\left(t_{1}, \ldots, t_{m}\right) \in T^{m}$ of reflections such that $w=t_{1} \cdots t_{m}$. This action is called Hurwitz action. A standard braid group generator $\sigma_{i}$ (resp. its inverse $\sigma_{i}^{-1}$ ) acts by a Hurwitz move on a reflection factorization:

$$
\begin{aligned}
\sigma_{i}\left(t_{1}, \ldots, t_{i-1}, t_{i}, t_{i+1}, t_{i+2}, \ldots, t_{n}\right) & =\left(t_{1}, \ldots, t_{i-1}, t_{i+1}^{t_{i}}, t_{i}, t_{i+2}, \ldots, t_{n}\right), \\
\sigma_{i}^{-1}\left(t_{1}, \ldots, t_{i-1}, t_{i}, t_{i+1}, t_{i+2}, \ldots, t_{n}\right) & =\left(t_{1}, \ldots, t_{i-1}, t_{i+1}, t_{i}^{t_{i+1}}, t_{i+2}, \ldots, t_{n}\right),
\end{aligned}
$$

where we use the notation $g^{h}:=h g h^{-1}$ for conjugation.

We call a reflection factorization $\left(t_{1}, \ldots, t_{m}\right)$ of an element $w \in W$ reduced if $w$ cannot be written as a product of less than $m$ reflections. It has been first observed by Deligne [3] that this action is transitive on reduced reflection factorizations of a Coxeter element if $W$ is finite. The first published proof is due to Bessis [2, Proposition 1.6.1]. Igusa and Schiffler showed that this statement is true for every Coxeter group [7, Theorem 1.4].

The question of how these results extend to non-reduced reflection factorizations has been first addressed by Lewis and Reiner.

TheOREM (Lewis-Reiner, [8, Theorem 1.1]). In a finite real reflection group, two reflection factorizations of a Coxeter element lie in the same Hurwitz orbit if and only if they share the same multiset of conjugacy classes.

Their proof makes heavy use of the following remarkable result for finite Coxeter groups.

Manuscript received 21st January 2019, revised 25th June 2019, accepted 24th September 2019. KEywords. Coxeter groups, Hurwitz action, reflection factorizations, Coxeter element. 
Lemma 1.1 (Lewis-Reiner, [8, Corollary 1.4]). Let $(W, S)$ be a finite Coxeter system and $w \in W$. Then every reflection factorization of $w$ lies in the same Hurwitz orbit of some reflection factorization $\left(t_{1}, \ldots, t_{m}\right)$ of $w$ such that $\left(t_{1}, \ldots, t_{\ell}\right)$ is a reduced reflection factorization of $w$ for some $\ell \leqslant m$ and

$$
t_{\ell+1}=t_{\ell+2}, t_{\ell+3}=t_{\ell+4}, \ldots, t_{m-1}=t_{m} .
$$

This result is proved by a case-by-case analysis and seems not to extend to infinite Coxeter groups in general. We give a case-free proof of a similar (but weaker) result for all Coxeter groups of finite rank (see Lemma 2.3). In this way, we obtain that the result of Lewis and Reiner extends to all Coxeter groups of finite rank which provides a positive answer to a question of Lewis and Reiner [8, Question 6.2].

TheOREM 1.2. Let $(W, S)$ be a Coxeter system of finite rank. Then two reflection factorizations of a Coxeter element in $W$ lie in the same Hurwitz orbit if and only if they share the same multiset of conjugacy classes.

\section{THE PROOF}

Throughout this note let $(W, S)$ be a Coxeter system of finite rank $n \in \mathbb{N}$ with set of reflections $T=\left\{w s w^{-1} \mid w \in W, s \in S\right\}$. All necessary definitions and facts about Coxeter groups we will use are covered by $[1,4,5,6]$.

A subgroup $W^{\prime}$ of $W$ is called a reflection subgroup if $W^{\prime}=\left\langle W^{\prime} \cap T\right\rangle$. Each reflection subgroup $W^{\prime}$ admits a canonical set of generators $\chi\left(W^{\prime}\right)$ such that $\left(W^{\prime}, \chi\left(W^{\prime}\right)\right)$ is a Coxeter system and the set of reflections for $\left(W^{\prime}, \chi\left(W^{\prime}\right)\right)$ is given by $W^{\prime} \cap T=$ $\bigcup_{w \in W^{\prime}} w \chi\left(W^{\prime}\right) w^{-1}$ (see [4, (3.3) Theorem]). A reflection subgroup of the form $\langle I\rangle$ for some $I \subseteq S$, is called parabolic subgroup.

Let $S=\left\{s_{1}, \ldots, s_{n}\right\}$. For each permutation $\pi$ of the numbers $\{1, \ldots, n\}$, the element $c=s_{\pi(1)} \cdots s_{\pi(n)}$ is called a Coxeter element. A Coxeter element of a parabolic subgroup is called parabolic Coxeter element.

We denote by $\ell_{S}\left(\right.$ resp. $\left.\ell_{T}\right)$ the length function on $W$ with respect to the generating set $S$ (resp. $T$ ).

Definition 2.1. We define the Bruhat graph of $(W, S)$ to be the directed graph $\Omega_{(W, S)}$ on vertex set $W$ with a directed edge from $x$ to $y$ if there exists $t \in T$ such that $y=x t$ and $\ell_{S}(x)<\ell_{S}(y)$.

Moreover, we denote by $\bar{\Omega}_{(W, S)}$ the corresponding undirected graph and for a subset $X \subseteq W$ we denote by $\Omega_{(W, S)}(X)$ the full subgraph of $\Omega_{(W, S)}$ on the vertex set $X$.

Note that $\bar{\Omega}_{(W, S)}$ is exactly the Cayley graph of $W$ with generating set $T$.

We use the notation $\left(t_{1}, \ldots, t_{m}\right) \sim\left(r_{1}, \ldots, r_{m}\right)$ to indicate that both tuples lie in the same orbit under the Hurwitz action.

The following fact is already part of the proof of [1, Proposition 2.2]. For sake of completeness we include a proof (which can also be found in the first author's Ph.D. thesis [9, Proposition 2.3.6])

Proposition 2.2. Let $w \in W$ and $t_{1}, t_{2} \in T$ with $t_{1} \neq t_{2}$ such that

$$
w \rightarrow w t_{1} \leftarrow w t_{1} t_{2}
$$

in $\Omega_{(W, S)}$. Then there exist $t_{1}^{\prime}, t_{2}^{\prime} \in\left\langle t_{1}, t_{2}\right\rangle \cap T$ with $\left(t_{1}, t_{2}\right) \sim\left(t_{1}^{\prime}, t_{2}^{\prime}\right)$ such that one of the following cases hold:

(1) $w \rightarrow w t_{1}^{\prime} \rightarrow w t_{1}^{\prime} t_{2}^{\prime}=w t_{1} t_{2}$;

(2) $w \leftarrow w t_{1}^{\prime} \leftarrow w t_{1}^{\prime} t_{2}^{\prime}=w t_{1} t_{2}$;

(3) $w \leftarrow w t_{1}^{\prime} \rightarrow w t_{1}^{\prime} t_{2}^{\prime}=w t_{1} t_{2}$;

Furthermore, in each of these cases we have $\ell_{S}\left(w t_{1}^{\prime}\right)<\ell_{S}\left(w t_{1}\right)$. 
Proof. Consider the dihedral reflection subgroup $W^{\prime}:=\left\langle t_{1}, t_{2}\right\rangle$ and let $S^{\prime}:=\chi\left(W^{\prime}\right)$. We have $w, w t_{1}, w t_{1} t_{2} \in w W^{\prime}$. Therefore we just have to consider the coset $w W^{\prime}$ to prove the claim. By $[5,(1.4)$ Theorem $]$ we have

$$
\Omega_{(W, S)}\left(W^{\prime}\right) \cong \Omega_{(W, S)}\left(w W^{\prime}\right) \cong \Omega_{\left(W^{\prime}, S^{\prime}\right)},
$$

where $\left(W^{\prime}, S^{\prime}\right)$ is dihedral and we can check the claim there directly. Any reflection (element of odd $S^{\prime}$-length) of $W^{\prime}$ is joined by an edge to a rotation (element of even $S^{\prime}$-length) which in $\Omega_{\left(W^{\prime}, S^{\prime}\right)}$ is oriented towards the element of greater $S^{\prime}$-length. For $x \in W^{\prime}$ there are three possible situations:

- $\ell_{S^{\prime}}(x)<\ell_{S^{\prime}}\left(x t_{1} t_{2}\right)$

- $\ell_{S^{\prime}}(x)>\ell_{S^{\prime}}\left(x t_{1} t_{2}\right)$

- $\ell_{S^{\prime}}(x)=\ell_{S^{\prime}}\left(x t_{1} t_{2}\right)$ (in particular $x \neq e$ since $t_{1} \neq t_{2}$ ).

We can choose $t_{1}^{\prime}, t_{2}^{\prime} \in W^{\prime} \cap T$ with $t_{1}^{\prime} t_{2}^{\prime}=t_{1} t_{2}$ in the three situations such that we have one of the following situations:

$$
\begin{aligned}
& \text { - } x \rightarrow x t_{1}^{\prime} \rightarrow x t_{1}^{\prime} t_{2}^{\prime} \\
& \text { - } x \leftarrow x t_{1}^{\prime} \leftarrow x t_{1}^{\prime} t_{2}^{\prime} \\
& \text { - } x \leftarrow x t_{1}^{\prime} \rightarrow x t_{1}^{\prime} t_{2}^{\prime}
\end{aligned}
$$

To see this, note that $x$ and $x t_{1} t_{2}$ are both either reflections or rotations. Therefore both are either of odd or even $S^{\prime}$-length. Thus $\ell_{S^{\prime}}(x)<\ell_{S^{\prime}}\left(x t_{1} t_{2}\right)$ implies $\ell_{S^{\prime}}(x)+2 \leqslant$ $\ell_{S^{\prime}}\left(x t_{1} t_{2}\right)$ and we find $t_{1}^{\prime}$ with $\ell_{S^{\prime}}(x)<\ell_{S^{\prime}}\left(x t_{1}^{\prime}\right)<\ell_{S^{\prime}}\left(x t_{1} t_{2}\right)$. By setting $t_{2}^{\prime}:=t_{1}^{\prime} t_{1} t_{2}$ we obtain $x \rightarrow x t_{1}^{\prime} \rightarrow x t_{1}^{\prime} t_{2}^{\prime}$ and $t_{1}^{\prime} t_{2}^{\prime}=t_{1} t_{2}$. The remaining cases are similar.

It is easy to see that the Hurwitz orbit of $\left(t_{1}, t_{2}\right)$ is the set of all pairs $\left(r_{1}, r_{2}\right)$ of reflections of $W^{\prime}$ such that $t_{1} t_{2}=r_{1} r_{2}$ (see also [1]). Hence we have $\left(t_{1}, t_{2}\right) \sim\left(t_{1}^{\prime}, t_{2}^{\prime}\right)$.

It remains to show that $\ell_{S}\left(w t_{1}^{\prime}\right)<\ell_{S}\left(w t_{1}\right)$ in each of the cases (1)-(3). Since the initial path is of the form $w \rightarrow w t_{1} \leftarrow w t_{1} t_{2}$, we have:

(i) $\ell_{S}(w)<\ell_{S}\left(w t_{1}\right)$, and

(ii) $\ell_{S}\left(w t_{1} t_{2}\right)<\ell_{S}\left(w t_{1}\right)$.

In case (1) we have an edge $w t_{1}^{\prime} \rightarrow w t_{1}^{\prime} t_{2}^{\prime}=w t_{1} t_{2}$, thus

$$
\ell_{S}\left(w t_{1}^{\prime}\right)<\ell_{S}\left(w t_{1}^{\prime} t_{2}^{\prime}\right)=\ell_{S}\left(w t_{1} t_{2}\right) \stackrel{(\mathrm{ii})}{<} \ell_{S}\left(w t_{1}\right) .
$$

In cases (2) and (3) we have an edge $w \leftarrow w t_{1}^{\prime}$, thus

$$
\ell_{S}\left(w t_{1}^{\prime}\right)<\ell_{S}(w) \stackrel{(\mathrm{i})}{<} \ell_{S}\left(w t_{1}\right) .
$$

LEMmA 2.3. Let $w \in W$ with $\ell_{S}(w)=m$ and $w=t_{1} \cdots t_{m+2 k}$ with $t_{i} \in T$ for $1 \leqslant i \leqslant m+2 k$ and some $k \in \mathbb{Z}_{\geqslant 0}$. Then there exists a braid $\sigma \in \mathcal{B}_{m+2 k}$ such that

$$
\sigma\left(t_{1}, \ldots, t_{m+2 k}\right)=\left(r_{1}, \ldots, r_{m}, r_{i_{1}}, r_{i_{1}}, \ldots, r_{i_{k}}, r_{i_{k}}\right) .
$$

Proof. We proceed by induction on $k$. The case $k=0$ is trivially satisfied. Therefore let $k \geqslant 1$ and assume that all factorizations in $\mathcal{B}_{m+2 k}\left(t_{1}, \ldots, t_{m+2 k}\right)$ consist of pairwise different factors. Consider the path of $\Omega_{(W, S)}$ starting in $e$ and ending in $w$ induced by $\left(t_{1}, \ldots, t_{m+2 k}\right)$. Then Proposition 2.2 allows us to replace successively the parts of the path of shape $\star \rightarrow \star \leftarrow \star$ by

$$
\star \rightarrow \star \rightarrow \star, \star \leftarrow \star \leftarrow \star, \text { or } \star \leftarrow \star \rightarrow \star
$$

only using the Hurwitz action. The latter is possible since the reflections of the factorizations in the Hurwitz orbit are pairwise different. Since by Proposition 2.2 each replacement reduces the sum of the length of the vertices, eventually we get after finitely many replacements a path of the form

$$
e \leftarrow t_{1}^{\prime} \leftarrow t_{1}^{\prime} t_{2}^{\prime} \leftarrow \ldots \leftarrow t_{1}^{\prime} t_{2}^{\prime} \cdots t_{p}^{\prime} \rightarrow t_{1}^{\prime} t_{2}^{\prime} \cdots t_{p}^{\prime} t_{p+1}^{\prime} \rightarrow \ldots \rightarrow t_{1}^{\prime} \cdots t_{m+2 k}^{\prime}=w
$$


with $t_{i}^{\prime} \in T$ for $1 \leqslant i \leqslant m+2 k$, that is, the path is first decreasing, then increasing. Since the path starts with $e$, it holds $p=0$ and therefore it has no decreasing part. Altogether the initial path can be transformed to

$$
e \rightarrow t_{1}^{\prime} \rightarrow t_{1}^{\prime} t_{2}^{\prime} \rightarrow \ldots \rightarrow t_{1}^{\prime} \cdots t_{m+2 k}^{\prime}=w
$$

by using the Hurwitz action. Since the length of $w$ is $m$ and $k \geqslant 1$, there cannot be an increasing path of length $m+2 k$, so we arrive at a contradiction. Thus there exists a factorization $\left(t_{1}^{\prime}, \ldots, t_{m+2(k-1)}^{\prime}, r_{i_{k}}, r_{i_{k}}\right)$ in $\mathcal{B}_{m+2 k}\left(t_{1}, \ldots, t_{m+2 k}\right)$. From the induction hypothesis follows

$$
\left(t_{1}^{\prime}, \ldots, t_{m+2(k-1)}^{\prime}\right) \sim\left(r_{1}, \ldots, r_{m}, r_{i_{1}}, r_{i_{1}}, \ldots, r_{i_{k-1}}, r_{i_{k-1}}\right)
$$

and the latter yields the assumption.

REMARK 2.4. Note that $\ell_{T}(w) \leqslant \ell_{S}(w)$ for all $w \in W$. Therefore the reflection factorization $w=r_{1} \cdots r_{m}$ obtained by Lemma 2.3 does not have to be a reduced reflection factorization. This is the main difference compared with the key argument Lemma 1.1 in the proof of Lewis and Reiner. However, by [1, Lemma 2.1] we have $\ell_{S}(w)=\ell_{T}(w)$ for an element $w \in W$ if and only if $w$ is a parabolic Coxeter element. Therefore, if $w$ is a parabolic Coxeter element, then Lemma 2.3 generalizes Lemma 1.1. In particular, a reflection factorization of a parabolic Coxeter element can be reduced by just using Hurwitz moves and deleting matching neighbors.

A proof of the following fact already implicitly appears in the proof of [8, Theorem 1.1].

Lemma 2.5. Let $t_{1}, \ldots, t_{n}, t \in T$. Then $\left(t_{1}, \ldots, t_{n}, t, t\right) \sim\left(t_{1}, \ldots, t_{n}, t^{w}, t^{w}\right)$ for all $w \in\left\langle t_{1}, \ldots, t_{n}\right\rangle$.

Proof. Let $i \in\{1, \ldots, n\}$ be arbitrary and assume $w=t_{i}$ (the general assertion follows by induction). Denoting an omitted entry by $\widehat{t_{i}}$, we obtain

$$
\begin{aligned}
\left(t_{1}, \ldots, t_{n}, t, t\right) & \sim\left(t_{1}, \ldots, t_{i-1}, \widehat{t_{i}}, t_{i+1}^{t_{i}} \ldots, t_{n}^{t_{i}}, t^{t_{i}}, t^{t_{i}}, t_{i}\right) \\
& \sim\left(t_{1}, \ldots, t_{i-1}, \widehat{t_{i}}, t_{i+1}^{t_{i}} \ldots, t_{n}^{t_{i}}, t_{i}, t^{t_{i}}, t^{t_{i}}\right) \\
& \sim\left(t_{1}, \ldots, t_{i-1}, t_{i}, t_{i+1}, \ldots, t_{n}, t^{t_{i}}, t^{t_{i}}\right) .
\end{aligned}
$$

Proof of Theorem 1.2. Let $c \in W$ be a Coxeter element and

$$
c=t_{1}^{\prime} \cdots t_{n+2 k}^{\prime}=r_{1}^{\prime} \cdots r_{n+2 k}^{\prime}
$$

two reflection factorizations of $c$ for some $k \in \mathbb{Z}_{\geqslant 0}$ such that they share the same multiset of conjugacy classes. By Lemma 2.3 we have

$$
\begin{aligned}
\left(t_{1}^{\prime}, \ldots, t_{n+2 k}^{\prime}\right) & \sim\left(t_{1}, \ldots, t_{n}, t_{i_{1}}, t_{i_{1}}, \ldots, t_{i_{k}}, t_{i_{k}}\right) \\
\text { and }\left(r_{1}^{\prime}, \ldots, r_{n+2 k}^{\prime}\right) & \sim\left(r_{1}, \ldots, r_{n}, r_{i_{1}}, r_{i_{1}}, \ldots, r_{i_{k}}, r_{i_{k}}\right) .
\end{aligned}
$$

Since $c=t_{1} \cdots t_{n}=r_{1} \cdots r_{n}$ and $\ell_{S}(c)=\ell_{T}(c)=n$ by $\left[1\right.$, Lemma 2.1], $\left(t_{1}, \ldots, t_{n}\right)$ and $\left(r_{1}, \ldots, r_{n}\right)$ are reduced reflection factorizations of $c$. Hence we have $\left(t_{1}, \ldots, t_{n}\right) \sim$ $\left(r_{1}, \ldots, r_{n}\right)$ by $\left[1\right.$, Theorem 1.3]. In particular $\left(t_{1}, \ldots, t_{n}\right)$ and $\left(r_{1}, \ldots, r_{n}\right)$ share the same multiset of conjugacy classes. Hence $t_{i_{1}}, \ldots, t_{i_{k}}$ and $r_{i_{1}}, \ldots, r_{i_{k}}$ have to share the same multiset of conjugacy classes. Since $(t, t, r, r) \sim(r, r, t, t)$ for all $r, t \in T$, we can assume after a possible renumbering that there exists $w_{j} \in W$ such that $t_{i_{j}}^{w_{j}}=r_{i_{j}}$ for all $j \in\{1, \ldots, k\}$. We proceed by induction on $k$. As we have seen above, the case $k=0$ is precisely [1, Theorem 1.3]. Therefore let $k>0$. By induction we have

$\left(t_{1}, \ldots, t_{n}, t_{i_{1}}, t_{i_{1}}, \ldots, t_{i_{k-1}}, t_{i_{k-1}}, t_{i_{k}}, t_{i_{k}}\right) \sim\left(r_{1}, \ldots, r_{n}, r_{i_{1}}, r_{i_{1}}, \ldots, r_{i_{k-1}}, r_{i_{k-1}}, t_{i_{k}}, t_{i_{k}}\right)$. 
As a consequence of [1, Theorem 1.3], we have $W=\left\langle r_{1}, \ldots, r_{n}\right\rangle$. By what we have pointed out before, there exists $w_{k} \in\left\langle r_{1}, \ldots, r_{n}\right\rangle$ such that $t_{i_{k}}^{w_{k}}=r_{i_{k}}$. We conclude

$$
\begin{aligned}
\left(t_{1}^{\prime}, \ldots, t_{n+2 k}^{\prime}\right) & \sim\left(r_{1}, \ldots, r_{n}, r_{i_{1}}, r_{i_{1}}, \ldots, r_{i_{k-1}}, r_{i_{k-1}}, t_{i_{k}}, t_{i_{k}}\right) \\
& \sim\left(r_{1}, \ldots, r_{n}, t_{i_{k}}, t_{i_{k}}, r_{i_{1}}, r_{i_{1}}, \ldots, r_{i_{k-1}}, r_{i_{k-1}}\right) \\
& \sim 2.5\left(r_{1}, \ldots, r_{n}, t_{i_{k}}^{w_{k}}, t_{i_{k}}^{w_{k}}, r_{i_{1}}, r_{i_{1}}, \ldots, r_{i_{k-1}}, r_{i_{k-1}}\right) \\
& =\left(r_{1}, \ldots, r_{n}, r_{i_{k}}, r_{i_{k}}, r_{i_{1}}, r_{i_{1}}, \ldots, r_{i_{k-1}}, r_{i_{k-1}}\right) \\
& \sim\left(r_{1}, \ldots, r_{n}, r_{i_{1}}, r_{i_{1}}, \ldots, r_{i_{k-1}}, r_{i_{k-1}}, r_{i_{k}}, r_{i_{k}}\right) \\
& \sim\left(r_{1}^{\prime}, \ldots, r_{n+2 k}^{\prime}\right) .
\end{aligned}
$$

COROLlary 2.6. If the Coxeter graph of $(W, S)$ is connected and has a spanning tree with odd labels on all its edges, then two reflection factorizations of the same length of a Coxeter element in $W$ lie in the same Hurwitz orbit.

Proof. Since the Coxeter graph of $(W, S)$ contains a spanning tree, all elements of $S$ are conjugate. Therefore all reflections in $T$ are conjugate. Thus the assertion follows by Theorem 1.2 .

Acknowledgements. The authors thank two anonymous referees for helpful comments.

\section{REFERENCES}

[1] Barbara Baumeister, Matthew Dyer, Christian Stump, and Patrick Wegener, A note on the transitive Hurwitz action on decompositions of parabolic Coxeter elements, Proc. Am. Math. Soc., Ser. B 1 (2014), 149-154.

[2] David Bessis, The dual braid monoid, Ann. Sci. Éc. Norm. Supér. (4) 36 (2003), no. 5, 647-683.

[3] Pierre Deligne, Letter to E. Looijenga, 1974, http://homepage.rub.de/christian.stump/ Deligne_Looijenga_Letter_09-03-1974.pdf (accessed 30th September 2019).

[4] Matthew Dyer, Reflection subgroups of Coxeter systems, J. Algebra 135 (1990), no. 1, 57-73.

[5] _ On the "Bruhat graph" of a Coxeter system, Compos. Math. 78 (1991), no. 2, 185-191.

[6] James E. Humphreys, Reflection groups and Coxeter groups, Cambridge Studies in Advanced Mathematics, vol. 29, Cambridge University Press, Cambridge, 1990.

[7] Kiyoshi Igusa and Ralf Schiffler, Exceptional sequences and clusters, J. Algebra 323 (2010), no. 8, 2183-2202.

[8] Joel Brewster Lewis and Victor Reiner, Circuits and Hurwitz action in finite root systems, New York J. Math. 22 (2016), 1457-1486.

[9] Patrick Wegener, Hurwitz action in Coxeter groups and elliptic Weyl groups, Ph.D. thesis, Universität Bielefeld (Germany), 2017, https://pub.uni-bielefeld.de/record/2913106.

Patrick Wegener, Technische Universität Kaiserslautern, Fachbereich Mathematik, Postfach 3049, 67653 Kaiserslautern, Germany

E-mail : wegener@mathematik.uni-kl.de

Sophiane Yahiatene, Universität Bielefeld, Fakultät für Mathematik, Postfach 100131, 33501

Bielefeld, Germany

E-mail : syahiate@math.uni-bielefeld.de 